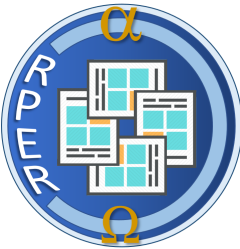

Revista Portuguesa de Enfermagem de Reabilitação

ISSN: 2184-3023

Vol. 4 N. ${ }^{\circ} 1(2021)$

Publicado 30-06-2021

Pag.: $64-72$

Artigo: Revisão de literatura

DOI: https://doi.org/10.33194/rper.2021.v4.n1.172

\title{
OXIGENOTERAPIA DE ALTO FLUXO POR TRAQUEOSTOMIA NO DESMAME VENTILATÓRIO: SCOPING REVIEW
}

\author{
HIGH-FLOW OXYGEN THERAPY FOR VENTILATOR WEANING OF TRACHEOSTOMIZED \\ PATIENTS: SCOPING REVIEW
}

\section{OXIGENOTERAPIA DE ALTO FLUJO EN LA DESCONEXIÓN DEL VENTILADOR DEL PACIENTE TRAQUEOSTOMIZADO: SCOPING REVIEW}

\author{
Sandrine Neiva ${ }^{(1)}$, Diana Maia ${ }^{(2)}$, Artur Pacheco ${ }^{(3)}$, Fernanda Marques ${ }^{(4)}$, Olga Ribeiro ${ }^{(5)}$, João Pedro Oliveira ${ }^{(6)}$,
}

(1) Centro Hospitalar Universitário de São João, Porto, Portugal; (2) Centro Hospitalar Universitário de São João, Porto, Portugal; (3) Centro Hospitalar Universitário de São João, Porto, Portugal; (4) Centro Hospitalar Universitário de São João, Porto, Portugal; (5) Escola Superior de Enfermagem do Porto (ESEP), Centro de Investigação em Tecnologias e Serviços de Saúde (CINTESIS), Porto, Portugal; (6) Centro Hospitalar Universitário de São João, Porto, Portugal;

\section{Descritores}

Oxigenoterapia

Traqueostomia

Enfermagem

Reabilitação

Desmame do Respirador

\section{Resumo}

Introducão: A ventilação mecânica invasiva prolongada acarreta custos e tem complicacõos associadas. Um desmame ventilatório precoce com sucesso, assume cada vez mais importância, pretendendo-se neste estudo descrever as evidências acerca da utilização da Oxigenoterapia de Alto Fluxo por Traqueostomia neste processo.

Metodologia: Scoping Review segundo a metodologia do Joanna Briggs Institute ${ }^{\circledR}$, com pesquisa efetuada nas bases de dados: CINHAL, PubMed e LILACS.

Resultados: Incluíram-se quatro estudos: um estudo de caso e três estudos controlados randomizados. Relativamente aos protocolos utilizados na implementação da oxigenoterapia de alto fluxo por traqueostomia, eles variaram entre diferentes fluxos, tempo de utilizacão e combinacão com outros modos ventilatórios. Os principais parâmetros monitorizados foram: frequência respiratória, $\mathrm{FiO}_{2}$ $\mathrm{SpO} 2, \mathrm{PaO} 2, \mathrm{PaCO} 2$ e a relação $\mathrm{PaO} 2 / \mathrm{FiO}^{2}$.

Discussão: A utilização de oxigenoterapia de alto fluxo por traqueostomia apresenta vantagens face à utilização de outros modos ventilatórios durante o processo de desmame ventilatório. Esta estratégia repercute-se na melhoria dos parâmetros monitorizados e na diminuição do tempo de desmame.

Conclusão: As principais contribuições da oxigenoterapia de alto fluxo por traqueostomia no desmame ventilatório são: melhoria da oxigenação, diminuição do esforço respiratório, aumento do volume corrente, diminuição do tempo de desmame e melhoria da eficácía da limpeza das vias aéreas.

Registo do protocolo (Open Science Framework): osf.io/2tkzm; DOI: 10.17605/OSF.IO/WMQJN.

\section{Abstract}

Introduction: Prolonged invasive mechanical ventilation is costly and has associated complications. Successful early ventilatory weaning is increasingly important, and the aim of this study is to describe the evidence about the use of High Flow Oxygen Therapy by Tracheostomy in this process.

Methodology: Scoping Review according to the Joanna Briggs Institute ${ }^{\circledR}$ methodology, with research carried out in the following databases: CINHAL, PubMed and LILACS.

Results: Four studies were included: one case study and three randomized controlled studies. Regarding the protocols used in the implementation of high flow oxygen therapy by tracheostomy, they varied between different flows, time of use and combination with other ventilation modes. The main parameters monitored were: respiratory rate, $\mathrm{FiO} 2, \mathrm{SpO} 2, \mathrm{PaO} 2, \mathrm{PaCO} 2$ and the $\mathrm{PaO} 2$ / FiO2 ratio.

Discussion: The use of high flow oxygen therapy by tracheostomy has advantages over the use of other ventilation modes during the weaning process. This strategy has an impact on the improvement of the monitored parameters and on the decrease in the time of ventilatory weaning.

Conclusion: The main contributions of high-flow oxygen therapy through tracheostomy in ventilatory weaning are: improved oxygenation, decreased respiratory effort, increased tidal volume, decreased 
weaning time and improved airway cleaning efficiency.

Protocol registration (Open Science Framework): osf.io/2tkzm; DOI: 10.17605/OSF.IO/WMQJN

\section{Descriptores}

Oxigenoterapia Traqueostomía Enfermería

Rehabilitación

Desconexión del Ventilador

\section{Resumen}

Introducción: La ventilación mecánica invasiva prolongada es costosa y tiene complicaciones asociadas. El éxito del destete ventilatorio temprano es cada vez más importante, y el objetivo de este estudio es describir la evidencia sobre el uso de la terapia de oxígeno de alto flujo por traqueotomía en este proceso.

Metodología: Scoping Review según la metodología Joanna Briggs Institute $\circledast$, con investigación realizada en las siguientes bases de datos: CINHAL, PubMed y LILACS.

Resultados: Se incluyeron cuatro estudios: un estudio de caso y tres estudios controlados aleatorios. En cuanto a los protocolos utilizados en la implementación de la oxigenoterapia de alto flujo por traqueotomía, estos variaron entre diferentes flujos, tiempo de uso y combinación con otros modos de ventilación. Los principales parámetros monitorizados fueron: frecuencia respiratoria, $\mathrm{FiO} 2, \mathrm{SpO} 2$, $\mathrm{PaO} 2, \mathrm{PaCO} 2$ y el cociente $\mathrm{PaO} 2$ / FiO2.

Discusión: El uso de la terapia de oxígeno de alto flujo por traqueotomía tiene ventajas sobre el uso de otros modos de ventilación durante el proceso de destete. Esta estrategia repercute en la mejora de los parámetros monitorizados y en la disminución del tiempo de destete ventilatorio.

Conclusión: Las principales contribuciones de la terapia de oxígeno de alto flujo por traqueotomía en el destete ventilatorio son: mejora de la oxigenación, disminución del esfuerzo respiratorio, aumento del volumen corriente, disminución del tiempo de destete y mejora de la eficiencia de limpieza de la vía aérea.

Registro de protocolo(Open Science Framework): osf.io/2tkzm; DOI: 10.17605/OSF.IO/WMQJN

\section{INTRODUÇÃO}

A Medicina Intensiva, é uma especialidade multidisciplinar e interprofissional, que está orientada para o cuidado e tratamento de pessoas, com ou em risco de desenvolver disfunção orgânica aguda, que implique risco de vida. Recorre a um conjunto de tecnologias de suporte à falência de vários sistemas corporais, particularmente, os sistemas pulmonar, cardiovascular e renal ${ }^{1}$.

Nas Unidades de Cuidados Intensivos (UCl), o desmame da Ventilação Mecânica Invasiva (VMI) é uma preocupação dos profissionais responsáveis pelos cuidados ao doente crítico. Cada vez que um Desmame Ventilatório (DV) é atrasado, os custos e o risco de complicações provenientes da VMI aumentam. Noutra perspetiva, um DV prematuro, também pode colocar em causa a seguranca do doente e aumentar o risco de reintubação. 0 tempo gasto neste procedimento, é responsável por $40 \%$ do tempo total do doente que está sob suporte ventilatório. Daí ser essencial otimizar o desmame da ventilação mecânica invasiva².

Para que o doente tenha condições de efetuar o DV adequado, é necessário que este apresente: nível de consciência, grau de colaboração, estabilidade hemodinâmica, boa perfusão tecidual, independência de vasopressores (doses baixas e estáveis são toleráveis) e ausência de insuficiência coronária descompensada ou arritmias com repercussão hemodinâmica. Além disso, deverá ter adequadas trocas gasosas $\left[\mathrm{PaO}_{2}\right.$ (Pressão Parcial de Oxigênio) $>60 \mathrm{mmHg}$ com FiO 2 (Fração Inspirada de Oxigênio) < 0,4 e PEEP (Pressão Expiratória Final Positiva) $\leq 5$ a $8 \mathrm{cmH}_{2} \mathrm{O}$ ], ser capaz de iniciar os esforços inspiratóriose evidenciar capacidade de mobilizar e eliminar as secreções ${ }^{3}$.

Uma forma de facilitar o DV ou evitar a falha deste, em doentes que requerem uma ventilação mecânica prolongada (VMP), é o uso da traqueostomia. Esta, para além de diminuir as complicações associadas à presença de um tubo endotraqueal de forma prolongada (pneumonia associada ao ventilador, sinusite, danos na traqueia e laringe), permite um acesso mais eficaz às vias aéreas e diminui a resistência destas; facilita o acesso mais eficaz e a eliminação de secreções brônquicas; reduz o trabalho respiratório e diminui a resistência das vias aéreas; melhora o conforto do doente, diminuindo a necessidade de sedação e promove a continuidade de cuidados dentro e fora da $\mathrm{UCl}^{4}$.

O desmame de doentes traqueostomizados, de uma VMP, não se traduz apenas num desafio, mas também em grandes custos financeiros, estando associada a mortalidade e morbilidade significativas, que aumentam com o período de VMI. Estes doentes, representam 6-10\% de todos aqueles que estão ventilados, consumindo 37 a $50 \%$ dos recursos de uma $\mathrm{UCI}^{5}$.

Mais recentemente, foi desenvolvida uma tecnologia que permite fornecer ao doente oxigénio, a partir de um sistema de alto fluxo por meio de cânula nasal, como meio alternativo à oxigenoterapia convencional. Esta foi denominada de Oxigenoterapia de Alto Fluxo por Cânula Nasal (ONAF), amplamente aceite, melhorando a hipóxia e o prognóstico dos doentes com Insuficiência Respiratória Aguda Hipoxémica (IRAH) ${ }^{2}$. Fornece ao doente concentrações de oxigénio mais elevadas, que as técnicas convencionais de oxigenoterapia ${ }^{6}$. Tem sido utilizada no período pós-extubação em doentes ventilados e após cirurgia cardiotorácica. Para além do fornecimento por cânula nasal, esta técnica de alto fluxo, pode ser implementada através de traqueostomia, assumindo a designação de Oxigenoterapia de Alto Fluxo por Traqueostomia (OTAF) ${ }^{7}$.

A ONAF, tem sido associada à menor necessidade de entubação endotraqueal, baixa mortalidade e à redução do risco de admissão e tempo de permanência nas $\mathrm{UCI}$, baixas taxas de reintubação por IRAH e redução de complicações relacionadas com a VM ${ }^{6}$. Fisiologicamente, esta aumenta o volume pulmonar expiratório final, devido a geracão de pressão positiva dependente dos fluxos utilizados. 0 alto fluxo contínuo (até $60 \mathrm{l} / \mathrm{min}$ ), com um $\mathrm{FiO}_{2}$ que pode variar de 21 a $100 \%$, por sua vez, promove a eliminação do dióxido de carbono das vias respiratórias superiores, reduzindo o espaço morto anatómico e o trabalho respiratório. A própria humidificação ( $\left.44 \mathrm{mg} \mathrm{H}_{2} \mathrm{O} / \mathrm{l}\right)$ e aquecimento (entre 31 e $37^{\circ} \mathrm{C}$ ) ativos e a interface confortável, melhoram o desconforto provocado pela secura das vias aéreas. Esta humidificação aumenta o conteúdo de água do muco, facilitando assim a remoção das secreções, evitando simultaneamente a dissecação e lesão epitelial, com o consequente aumento do conforto para o doente ${ }^{8}$.

Frequentemente utilizada nos últimos anos e com sucesso no desmame de doentes sob VMI, a ONAF tem-se mostrado mais eficaz, quando comparada, com a oxigenoterapia convencional e com a Ventilação Não Invasiva (VNI) ${ }^{4}$. Embora mais recentemente, a OTAF já mostrou 
que melhora o trabalho respiratório, que constitui um auxilio à ventilação e oxigenação, podendo ser útil no DV ${ }^{9}$. No entanto, faltam ainda evidências seguras acerca do seu contributo por traqueostomia, de forma isolada ou combinada com a VNI, na facilitação do processo de DV em pessoas traqueostomizadas 4 .

Neste sentido, definiu-se como objetivo deste estudo, descrever a evidência existente sobre o uso da OTAF no desmame ventilatório.

\section{METODOLOGIA}

Este estudo é uma Scoping Review, que seguiu as recompensações do Joanna Briggs Institute Reviewer's Manual, caracterizando-se por fazer um mapeamento da evidência preliminar existente sobre um determinado assunto ${ }^{10}$. É portanto, uma primeira análise da evidência emergente, quando esta ainda não está claramente apresentada, destinando-se a identificar a natureza e a abrangência das potenciais evidências $^{11}$.

Na redação do estudo, foi utilizado o instrumento PRISMA Extension for Scoping Reviews (PRISMA-SCR) ${ }^{12}$.

A pesquisa foi efetuada em 3 bases de dados: CINHAL, PubMed e LILACS, tendo sido realizada no mês abril de 2021, com um espaço temporal de janeiro de 2016 a abril de 2021. A opção por este espaço temporal esteve relacionada com o investimento efetuado nos últimos anos em relação ao uso da oxigenoterapia de alto fluxo por traqueostomia no desmame ventilatório. Os critérios de inclusão utilizados foram: estudos em texto completo e acesso gratuito nas bases de dados referidas, nos idiomas de português, inglês e espanhol e que tinham como população/amostra em estudo adultos traqueostomizados em desmame ventilatório. Foram excluídos documentos como cartas ao editor, resumos de conferências e estudos que não apresentavam como objeto de estudo a oxigenoterapia de alto fluxo por traqueostomia durante o desmame ventilatório.

Para a definição da pergunta de pesquisa utilizou-se a estratégia PCC (Quadro 1), tendo-se definido a seguinte questão: “Quais as evidências disponíveis acerca da utilização da Oxigenoterapia de Alto Fluxo por Traqueostomia no Desmame Ventilatório?”.

Quadro 1 - Estratégia PCC

\begin{tabular}{l|l}
$\mathrm{P}$ (Population) & Doentes traqueostomizados em desmame ventilatório \\
$\mathrm{C}($ Concept) & Oxigenoterapia de alto fluxo por traqueostomia \\
\hline $\mathrm{C}$ (Context) & Contexto Hospitalar
\end{tabular}

Para a realização da pesquisa nas bases de dados, os descritores utilizados foram extraídos do vocabulário DeCS/MeSH (Descritores em Ciências da Saúde): 1. Oxigenoterapia; 2. Traqueostomia; 3. Desmame do Respirador; 4. Enfermagem; 5 . Reabilitação (Português) ou 1. Oxygen Inhalation Therapy; 2.Tracheostomy; 3. Ventilator Weaning; 4. Nursing; 5. Rehabilitation (Inglês). Para a conjugação dos descritores foram utilizados de forma lógica o operador booleano e truncador: AND e *.

Posteriormente, para extracão dos dados dos artigos selecionados, foi elaborado um protocolo. Foi realizado o registo do protocolo na Open Science Framework a 22 de junho de 2021 sendo atribuído o número osf.io/2tkzm e o seguinte DOI, 10.17605/OSF.IO/WMQJN. Para além da estratégia de pesquisa, o protocolo apresenta a estratégia de extração de dados que agrega os seguintes aspetos: país; ano de publicação; objetivo do estudo; tipo de estudo; critérios de elegibilidade; local de realizacão da intervenção; população; métodos para a implementação da intervenção; medidas utilizadas para avaliar a intervenção; níveis de evidência e resultados.

De modo a responder à questão de pesquisa, dos artigos selecionados, foram extraídas as seguintes informações: 1) título do artigo, 2) tipo de estudo, 3) objetivos e 4) resultados.

Importa referir que durante o processo de análise dos estudos, dois revisores independentes procederam à avaliação crítica, extração e síntese dos dados, sendo que em caso de discordância, se recorreu a um terceiro revisor.

Os estudos foram classificados quanto aos níveis de evidência, de acordo com o Instituto Joanna Briggs ${ }^{10}$ e os resultados foram apresentados de forma descritiva, com recurso a tabelas, figuras e gráficos.

\section{RESULTADOS}

Dos 49 estudos encontrados nas diferentes bases de dados foram incluídos neste estudo 4 artigos (Figura 1).

Dos quatro estudos incluídos, três são ensaios clínicos controlados randomizados (A1, A3, A4) e um estudo de caso (A2). Estes estudos foram realizados entre 2016 e 2020, em países da Europa, Japão e Austrália. Nos diferentes grupos o total das amostras foram 378 doentes (Figura 2).

0 quadro 2 caracteriza os estudos incluídos de acordo com o país de origem, ano de publicação, tipo de estudo, principais objetivos e resultados, bem como os níveis de evidência de acordo com o Joanna Briggs Institute ${ }^{10}$.

No Quadro 3 podem consultar-se o contexto da intervenção e as metodologias utilizadas. 


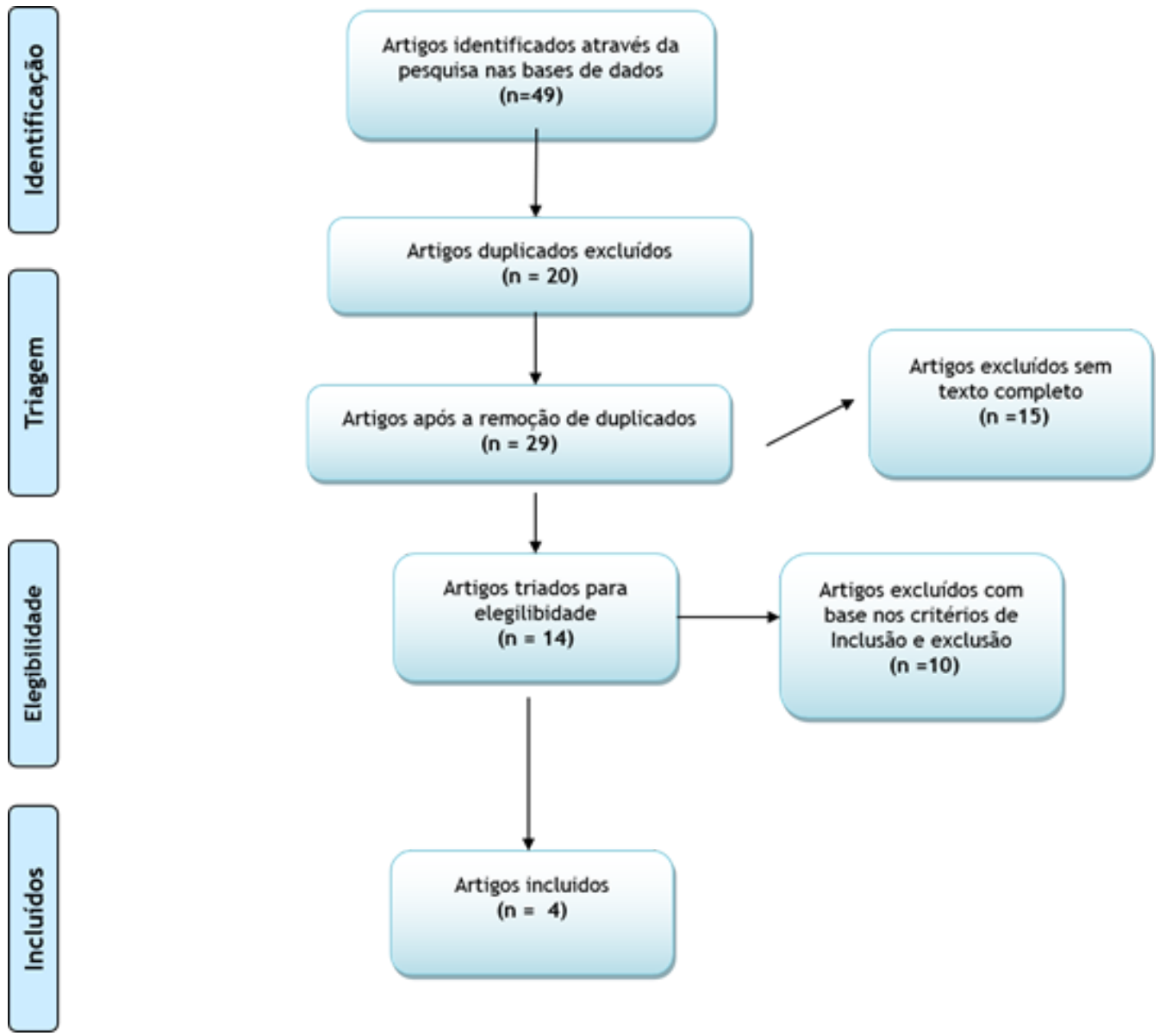

Figura 1 - Prisma SCR Flow diagram para a Scoping Review sobre oxigenoterapia de alto fluxo por traqueostomia no desmame ventilatório

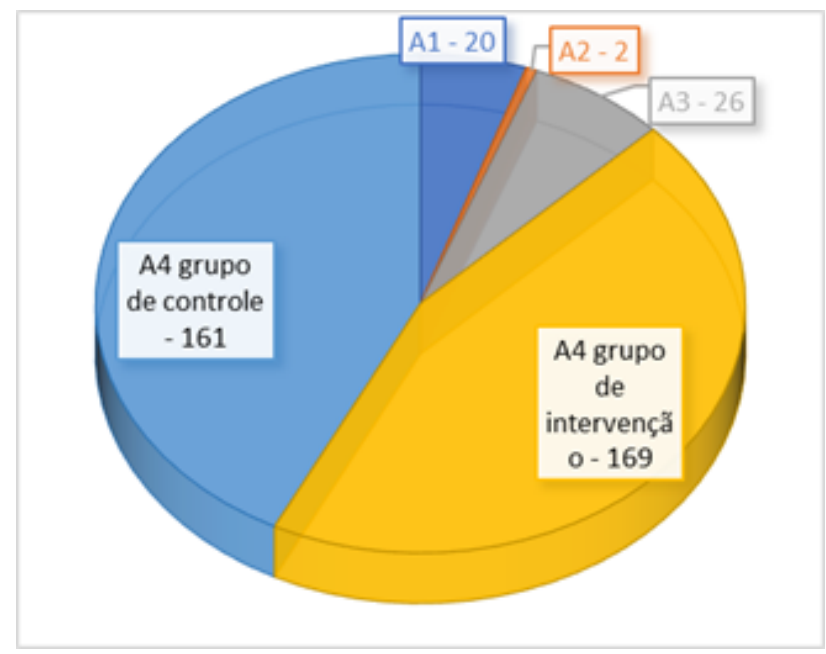

Figura 2 - Número de participantes nos estudos incluídos

Quadro 2 - Características dos estudos

\begin{tabular}{|c|c|c|c|c|c|c|c|}
\hline Artigo & $\begin{array}{l}\text { Título do artigo } \\
\text { (resumo avaliado) }\end{array}$ & $\begin{array}{l}\text { Tipo de } \\
\text { estudo }\end{array}$ & Ano & País & Objetivo & Resultados & $\begin{array}{l}\text { Nivel de } \\
\text { Evidência }\end{array}$ \\
\hline $\mathrm{A} 1^{13}$ & $\begin{array}{l}\text { High-flow oxygen via } \\
\text { tracheostomy } \\
\text { improves oxygenation in } \\
\text { patients weaning from } \\
\text { mechanical ventilation: } \\
\text { a randomised crossover } \\
\text { study }\end{array}$ & $\begin{array}{l}\text { Ensaio clinico } \\
\text { controlado } \\
\text { randomizado }\end{array}$ & 2017 & Austrália & $\begin{array}{l}\text { Demonstrar que a } \\
\text { OAF melhora a } \\
\text { oxigenação em } \\
\text { doentes } \\
\text { traqueostomizados } \\
\text { em DV. }\end{array}$ & $\begin{array}{l}\text { A OAF pode ser útil para } \\
\text { aumentar a oxigenação } \\
\text { durante o DV, no entanto, o } \\
\text { desvio da laringe e das vias } \\
\text { aéreas superiores pode anular } \\
\text { alguns dos seus efeitos } \\
\text { benéficos. Conclui-se ainda } \\
\text { que são necessários mais } \\
\text { ensaios clínicos controlados } \\
\text { para se comprovar que a OAF } \\
\text { é útil para reduzir o tempo de }\end{array}$ & $1 \mathrm{C}$ \\
\hline
\end{tabular}




\begin{tabular}{|c|c|c|c|c|c|c|c|}
\hline & & & & & & $\begin{array}{l}\text { ventilação mecânica e } \\
\text { diminuir dias de internamento } \\
\text { em UCI. }\end{array}$ & \\
\hline$A 2^{14}$ & $\begin{array}{l}\text { High-flow oxygen via } \\
\text { tracheostomy } \\
\text { facilitates weaning from } \\
\text { prolonged } \\
\text { mechanical ventilation } \\
\text { in patients with } \\
\text { restrictive pulmonary } \\
\text { dysfunction: } \\
\text { two case reports }\end{array}$ & Estudo de caso & 2018 & Japão & $\begin{array}{l}\text { Verificar se a } \\
\text { utilização da OAF } \\
\text { facilita o desmame } \\
\text { ventilatório em } \\
\text { doentes } \\
\text { traqueostomizados } \\
\text { com patologia } \\
\text { pulmonar restritiva. }\end{array}$ & $\begin{array}{l}\text { A OTAF reduz o esforço } \\
\text { inspiratório e aumenta o } \\
\text { volume corrente quando } \\
\text { comparada com oxigénio por } \\
\text { peça em T, facilitando desta } \\
\text { forma o DV em doentes com } \\
\text { patologia pulmonar restritiva. }\end{array}$ & $4 \mathrm{D}$ \\
\hline$A 3^{15}$ & $\begin{array}{l}\text { Physiological efects of } \\
\text { high-fow oxygen } \\
\text { in tracheostomized } \\
\text { patients }\end{array}$ & $\begin{array}{l}\text { Ensaio clinico } \\
\text { controlado } \\
\text { randomizado }\end{array}$ & 2019 & Itália & $\begin{array}{l}\text { Demonstrar que a } \\
\text { OAF em doentes } \\
\text { traqueostomizados } \\
\text { apresenta benefício } \\
\text { fisiológico ao nível da } \\
\text { ventilação. }\end{array}$ & $\begin{array}{l}\text { Em comparação com a } \\
\text { oxigenoterapia nasal } \\
\text { convencional, em OTAF é } \\
\text { necessário um fluxo de } \\
50 l / \text { min para melhorar a } \\
\text { oxigenação, reduzir a } \\
\text { frequência respiratória e } \\
\text { fornecer pressão expiratória } \\
\text { positiva nas vias aéreas. }\end{array}$ & $1 \mathrm{C}$ \\
\hline$A 4^{16}$ & $\begin{array}{l}\text { High-Flow Oxygen with } \\
\text { Capping or Suctioning } \\
\text { for Tracheostomy } \\
\text { Decannulation. }\end{array}$ & $\begin{array}{l}\text { Ensaio clinico } \\
\text { controlado } \\
\text { randomizado }\end{array}$ & 2020 & Espanha & $\begin{array}{l}\text { Comprovar que a OAF } \\
\text { contínua é mais } \\
\text { eficaz do que a } \\
\text { oxigenoterapia de } \\
\text { alto fluxo } \\
\text { intermitente nos } \\
\text { doentes } \\
\text { traqueostomizados } \\
\text { em processo de } \\
\text { descanulação. }\end{array}$ & $\begin{array}{l}\text { Os doentes do grupo de } \\
\text { intervenção têm mais } \\
\text { benefício ao receber terapia } \\
\text { de OAF contínuo do que os } \\
\text { doentes no grupo de controle. } \\
\text { Basear a decisão de } \\
\text { descanulação na frequência } \\
\text { de aspiração e na terapia de } \\
\text { oxigênio de alto fluxo } \\
\text { contínuo, em vez de testes de } \\
\text { limitação de } 24 \text { horas e } \\
\text { terapia de oxigênio de alto } \\
\text { fluxo intermitente, reduziu o } \\
\text { tempo de descanulação, sem } \\
\text { evidência de } \\
\text { diferença entre os grupos na } \\
\text { incidência de falha de } \\
\text { descanulação. }\end{array}$ & $1 \mathrm{C}$ \\
\hline
\end{tabular}

Quadro 3 - Contexto de intervenção e metodologias

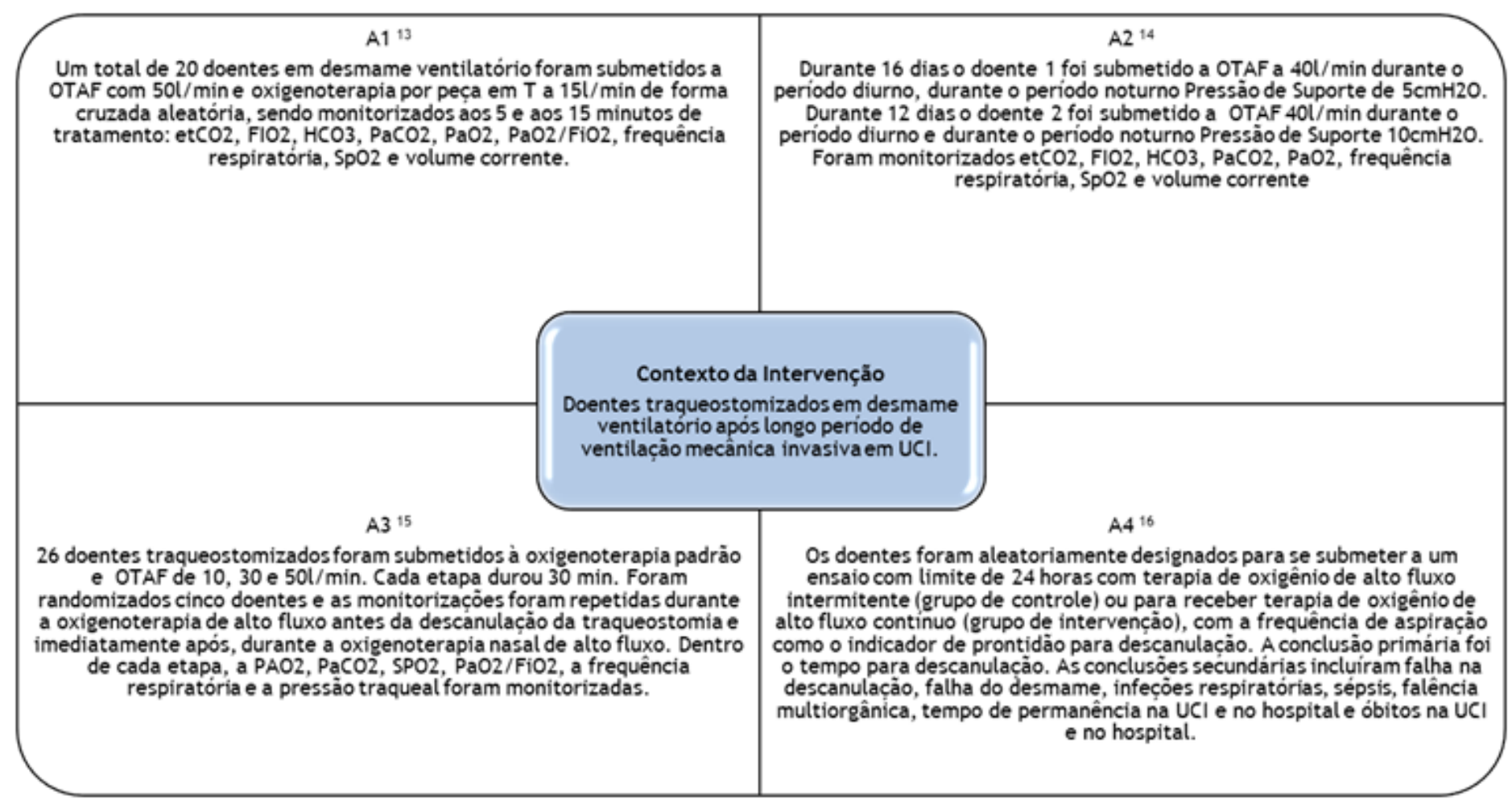

Observou-se que em todos os estudos o objetivo principal era averiguar o efeito da OTAF no processo do DV em contexto de cuidados intensivos. 
Relativamente aos fluxos de oxigénio utilizados eles variaram entre os $10 \mathrm{l} / \mathrm{min}$ e os $50 \mathrm{l} / \mathrm{min}$, sendo a sua utilização intermitente ou contínua $^{13,14,15}$. Durante o processo de DV, verificou-se que num estudo foi utilizada pressão de suporte noturna ${ }^{14}$ e outro oxigenoterapia convencional ${ }^{13}$.

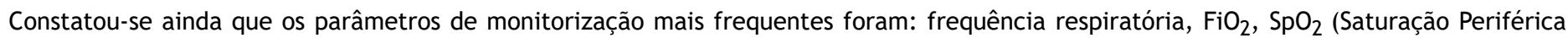
de Oxigénio), $\mathrm{PaO}_{2}$ e a $\mathrm{PaCO}_{2}$ (Pressão Parcial de Dióxido de Carbono) ${ }^{13,14,15}$.

Num dos estudos controlados randomizado ${ }^{15}, 26$ doentes traqueostomizados foram submetidos à oxigenoterapia padrão e à OAF com fluxo de 10, 30 e $50 \mathrm{l} / \mathrm{min}$. Cada etapa durou 30 minutos. Foram randomizados cinco doentes e as monitorizações repetidas durante a OAF antes da descanulação da traqueostomia e, imediatamente após, durante a oxigenoterapia nasal de alto fluxo. Tal como em outros estudos, a $\mathrm{PaO2}$, $\mathrm{PaCO} 2$, SpO2, relação $\mathrm{PaO2} / \mathrm{FiO2}$, a frequência respiratória e a pressão traqueal foram monitorizadas dentro de cada etapa. No presente estudo, demonstrou-se que em comparação com o oxigênio padrão, OTAF atenua o balanço negativo na pressão das vias aéreas durante a inspiração, e quando o fluxo é estabeleçido em $50 \mathrm{l} / \mathrm{min}$, melhora a oxigenação e reduz levemente a frequência respiratória. Tal facto também foi comprovado em outros estudos incluídos na revisão ${ }^{(14,16)}$, onde osautores referem que apesar de faltarem dados sobre os benefícios clínicos da OTAF, um fluxo de $50 \mathrm{l} / \mathrm{min}$, melhora a oxigenação, reduz a frequência respiratória, fornece um pequeno grau de pressão expiratória positiva nas vias aéreas, diminui o número de aspirações de secreções e melhora o transporte mucociliar.

A OAF mostrou ser vantajosa em relação à oxigenoterapia convencional no que diz respeito à diminuição do tempo de DV e consecutiva descanulação ${ }^{15}$. Houve ainda um estudo controlado randomizado que ao comparar a OAF contínua e OAF intermitente demonstrou que há maior sucesso na utilização da OAF contínua por diminuir consideravelmente o tempo de descanulação da traqueostomia ${ }^{16}$.

Um outro estudo controlado randomizado com 20 doentes traqueostomizados ${ }^{13}$ demonstrou os efeitos da OAF a $50 \mathrm{l} / \mathrm{min}$ por traqueostomia no volume pulmonar expiratório final, volume corrente, pressão das vias aéreas, oxigenação (relação PaO2 / FiO2), ventilação [dióxido de carbono expirado (etCO2)], frequência respiratória, frequência cardíaca e dispneia subjetiva em comparação com oxigênio convencional a 15l/min através de peça em T. A relação PaO2 / FiO2 melhorou significativamente com OTAF em comparação com peça em T. A pressão das vias aéreas durante OÁF foi significativamente maior 15 minutos após o início da OAF do que em peça ém T, melhorando desta forma os níveis de oxigenação e facilitando o processo de DV. Neste estudo a frequência respiratória e etCO2 mantiveram-se inalterados durante a OTAF, indicando que a resistência das vias aéreas e o trabalho respiratório também não se alterou ${ }^{13}$.

No estudo de caso $^{14}$ com dois doentes traqueostomizados com disfunção pulmonar restritiva onde foi aplicado OTAF, os autores relataram um DV bem-sucedido. Neste estudo um dos doentes após longo período de ventilação com pressão de suporte foi submetido a OTAF com fluxo inicial de $40 \mathrm{l} / \mathrm{min}$ por traqueostomia durante 16 dias até ao fluxo final de $15 \mathrm{l} / \mathrm{min}$ e o outro doente foi submetido a OTAF a $40 \mathrm{l} / \mathrm{min}$ durante 12 dias até ao fluxo final de $20 \mathrm{l} / \mathrm{min}$. Tal como no estudo anterior foram monitorizados os seguintes parâmetros: relação $\mathrm{PaO} 2 / \mathrm{FiO} 2, \mathrm{PaO} 2, \mathrm{PaCO} 2$, Volume corrente, etCO2 e frequência respiratória, tendo-se concluído que as pressões das vias aéreas foram continuamente positivas e não se tornaram negativas mesmo durante a inspiração, sugerindo que o oxigénio de alto fluxo por traqueostomia reduz o esforço inspiratório. A PaCO2 e frequência respiratória foram gradualmente reduzindo durante a OTAF, enquanto o volume corrente aumentou gradualmente.

\section{DISCUSSÃO}

Verificou-se que a preocupação geral dos estudos era o impacto da OTAF no DV. A escolha correta do método utilizado para o DV terá efeito no seu sucesso e tempo necessário para o mesmo².

A realização de traqueostomia tem sido apontada por vários autores como facilitadora do desmame da ventilação mecânica invasiva devido à diminuição do trabalho respiratório, com decréscimo significativo da dispneia, frequência cardíaca e respiratória ${ }^{17}$. Esta conclusão vai ao encontro dos resultados verificados pelos estudos analisados ${ }^{13,14,15}$

Observou-se que a maioria dos estudos conclui que a utilização de OTAF é benéfica para o DV 13,14,15. Apesar de ainda existirem poucos estudos em relação à OTAF, constatou-se que para a ONAF já existem várias pesquisas que suportam a sua utilização nesta fase ${ }^{18}$. A OAF pode reduzir o tempo necessário para o desmame ventilatório, reduzindo também o tempo necessário para passar o teste de respiração espontânea e diminuir a taxa de reintubação².

Efetivamente, em todos os estudos há evidência da eficácia da utilização da OAF no DV em doentes traqueostomizados, sendo que em 3 artigos são demonstrados efeitos benéficos quer a nível da oxigenação, volume corrente, pressão expiratória nas vias aéreas, frequência respiratória e esforço inspiratório ${ }^{13,14,15}$. Outro estudo refere também que a OAF melhora a oxigenação e previne atelectasias ${ }^{19}$.

Relativamente aos protocolos utilizados, eles variaram entre diferentes fluxos e tempo de utilização. Quanto ao fluxo, observou-se que fluxos de oxigénio entre os $40 \mathrm{l} / \mathrm{min}$ e os $50 \mathrm{l} / \mathrm{min}$ apresentam melhores resultados durante este processo ${ }^{13,15}$. Verificou-se também que a utilização de OAF, isolada ou associada, a outros modos ventilatórios, ainda não é consensual ${ }^{20,21}$. A implementação de protocolos ajuda a diminuir o tempo de desmame e aumenta o seu sucesso ${ }^{22}$.

Ficou evidente que a pressão nas vias aéreas inferiores é maior e mais variável quando fluxos elevados são administrados através de cânula nasal do que por traqueostomia ${ }^{15}$. Isto sugere que o mecanismo de criação de pressão expiratória durante o alto fluxo de oxigênio não depende apenas do fluxo, mas também da maior resistência oferecida pelas vias aéreas superiores e fluxo expiratório do doente. Em doentes traqueostomizados, a resistência é limitada e a pressão é mínima ${ }^{3,15}$.

Outro fator que pode contribuir para o sucesso do DV e posterior remocão de traqueostomia é a presença de secrecões e o número de vezes que o doente é aspirado. Num dos estudos analisados, um protocolo baseado no número de aspirações de secreções e OAF contínuo, contribuiu para um desmame seguro ${ }^{16}$. Uma das particularidades da OAF é a humidificação e aquecimento do oxigénio fornecido ao doente. Quando comparado com sistemas não aquecidos, verificou-se que o aquecimento contribuiu para diminuir a frequência e a necessidade da aspiração de secreções ${ }^{23}$.

Além da estratégia utilizada na OTAF durante o DV vários autores sugerem que a realização concomitante de programas de reabilitação

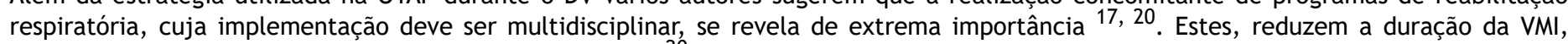
melhoram o conforto e facilitam a alta precoce do doente 20 .

Neste sentido, este processo é facilitado pela presença de enfermeiros de reabilitação nas unidades de cuidados intensivos, nomeadamente através do desenvolvimento de planos de cuidados individuais baseados numa crescente produção científica ${ }^{24}$. Programas 
de reabilitação e mobilização precoce em unidades de cuidados intensivos por enfermeiros de reabilitação revelaram-se seguros e com ganhos em saúde, nomeadamente na prevenção de complicações associadas à imobilidade ${ }^{25}$.

Encontra-se demonstrada a eficácia e necessidade da reabilitação respiratória durante este processo, nomeadamente no sucesso do DV 26 . Por outro lado, a ventilação mecânica prolongada poderá ter implicações neurológicas a longo prazo sendo necessário programas de reabilitação mais intensos e prolongados 27 .

\section{CONCLUSÃO}

Esta revisão permitiu concluir que a oxigenoterapia de alto fluxo por traqueostomia contribui para o sucesso e diminuição do tempo do desmame ventilatório, respondendo à nossa questão de pesquisa.

As principais contribuições da oxigenoterapia de alto fluxo por traqueostomia no desmame ventilatório são: melhoria da oxigenação, diminuição do trabalho respiratório, aumento do volume corrente, diminuição do tempo de desmame e melhoria da eficácia da limpeza das vias aéreas.

A utilização de protocolos para o desmame também se revelou essencial. A utilização de fluxos de oxigénio entre os 40-50l/min, a humidificação e o aquecimento facilitam o desmame ventilatório.

A presença de enfermeiros de reabilitação com a implementação de programas de reabilitação respiratória individualizados durante este processo contribui para o sucesso do mesmo.

A principal limitação deste estudo foi o número reduzido de artigos publicados sobre a temática. A OTAF sendo uma abordagem recente de utilização no desmame da VMI, trouxe-nos algumas dificuldades/ limitações, mesmo assim os resultados obtidos foram satisfatórios.

Consideramos de extrema relevância a realização de novos estudos, bem como, associando outras intervenções, nomeadamente programas de reabilitação respiratória e reeducação funcional motora.

\section{DIVULGAÇÕES ÉTICAS}

Contribuição do(s) autor(es):

Conceptualização: JO, AP, SN, FM, DM;

Metodologia: DM, OR;

Validação: JO, OR;

Análise formal: DM, SN, AP, FM;

Investigação: DM, SN, AP, FM;

Tratamento de dados: DM, SN, AP, FM;

Preparação do rascunho original: DM, SN, AP, FM;

Redação e edição: JO, AP;

Revisão: JO, OR;

Todos os autores leram e concordaram com a versão publicada do manuscrito.

Financiamento:

Este trabalho não recebeu nenhuma contribuição financeira ou bolsa.

Conflitos de interesse:

Os autores não declaram nenhum conflito de interesses.

Proveniência e revisão por pares:

Não comissionado; revisto externamente por pares.

\section{Referências}

1. Marshall JC, Bosco L, Adhikari NK, Connolly B, Diaz JV, Dorman T, et al. What is an intensive care unit? A report of the task force of the World Federation of Societies of Intensive and Critical Care Medicine. J. crit. care. [Internet]. 2017 [cited 2021 May 01];37:270-276. Doi: https://doi.org/10.1016/j.j.jcrc.2016.07.015

2. Liu F, Shao Q, Jiang R, Zeng Z, Liu Y, Li Y, et al. High-Flow Oxygen Therapy to Speed Weaning From Mechanical Ventilation: A Prospective Randomized Study. Am J Crit Care [Internet]. 2019 [cited 2021 May 01];28(5):370-376. Doi: https://doi.org/10.4037/ajcc2019130 
3. Goldwasser R, Farias A, Freitas EE, Saddy F, Amado V, Okamoto VN. Desmame e interrupcão da ventilação mecânica. Rev. bras. ter. intensiva [Internet]. 2007 [cited 2021 May 02];19(3):384-392. Doi: https: //doi.org/10.1590/S1806-37132007000800008

4. Guia M, Ciobanu LD, Sreedharan JK, Abdelrahim ME, Gonçalves G, Cabrita B, et al. The role of non-invasive ventilation in weaning and decannulating critically ill patients with tracheostomy: A narrative review of the literature. Pulmonology [Internet]. 2021 [cited 2021 Apr 26];27(1):43-51. Doi: https://doi.org/10.1016/j.pulmoe.2020.07.002

5. Füssenich W, Hirschfeld Araujo S, Kowald B, Hosman A, Auerswald M, Thietje R. Discontinuous ventilator weaning of patients with acute SCl. Spinal Cord [Internet]. 2018 [cited 2021 Apr 26];56(5):461-468. Doi: https://doi.org/10.1038/s41393-017-0055-x

6. Kaya A, Öz M, Erol S, Çiftçi F, Çileda? A, Kaya A. High flow nasal cannula in COVID-19: A literature review. Tuberk Toraks [Internet]. 2020 [cited $2021 \mathrm{Apr} 29] ; 68(2): 168$-174. Doi: https://doi.org/10.5578/tt.69807

7. Stripoli T, Spadaro S, Mussi R, Volta CA, Trerotoli P,Carlo F,et al. High-flow oxygen therapy in tracheostomized patients at high risk of weaning failure. Ann Intensive Care. [Internet]. 2019 [cited 2021 Apr 25];9(1):4. Doi: https://doi.org/10.1186/s13613-019-0482-2

8. Gilardi E, Petrucci M, Sabia L, Sellasie K, Grieco DL, Pennisi MA. High-flow nasal cannula for body rewarming in hypothermia. Crit Care [Internet]. 2020 [cited 2021 Apr 27];24(1):122. Doi: https://doi.org/10.1186/s13054-020-2839-1

9. Epstein D, Miller A, Ben-Avi R, Matan M. High-flow tracheal oxygen in tracheostomised COVID-19 patients. J Crit Care [Internet]. 2020 [cited 2021 Apr 29];59:35-36. Doi: https://doi.org/10.1016/j.jcrc.2020.05.015

10. The Joanna Briggs Institute. The Joanna Briggs Institute reviewers' manual 2015: Methodology for JBI scoping reviews [Internet]. Adelaide: The Joanna Briggs Institute; 2015. Available from: https://nursing. Isuhsc.edu/JBI/docs/ReviewersManuals/Scoping-.pdf

11. Sousa LM, Firmino CF, Marques-Vieira CM, Severino SS, Pestana HC. Revisões da Literatura Científica: Tipos, Métodos e Aplicações em Enfermagem. Rev Port Enf Reab [Internet]. 2018 [cited 2021 Apr 29];1(1):45-54. Available from: https://rper.aper.pt/index.php/rper/article/view/20

12. Tricco, AC, Lillie, E, Zarin, W, O'Brien, KK, Colquhoun, H, Levac, D, et al. PRISMA extension for scoping reviews (PRISMA-ScR): checklist and explanation. Ann Intern Med [Internet]. 2018 [cited 2021 May 02];169(7):467-473. Doi: https://doi.org/10.7326/M18-0850

13. Corley A, Edwards M, Spooner AJ, Dunster KR, Anstey C, Fraser JF. High-flow oxygen via tracheostomy improves oxygenation in patients weaning from mechanical ventilation: a randomised crossover study. Intensive care medicine [Internet]. 2017 Mar [cited 2021 May 1];43(3):465-7. Available from: http://search.ebscohost.com/login. aspx?direct=true\&db=mdc\&AN=27904928\&tang=pt-br\&site=ehost-live

14. Mitaka C, Odoh M, Satoh D, Hashiguchi T, Inada E. High-flow oxygen via tracheostomy facilitates weaning from prolonged mechanical ventilation in patients with restrictive pulmonary dysfunction: two case reports. J Med Case Rep [Internet]. 2018 [cited 2021 May 1];12(1):292. Available from: https://jmedicalcasereports.biomedcentral.com/articles/10.1186/s13256-018-1832-7

15. Natalini D, Grieco DL, Santantonio MT, Mincione L, Toni F, Anzellotti GM, et al. Physiological effects of high-flow oxygen in tracheostomized patients. Ann Intensive Care [Internet]. 2019 [cited 2021 May 3];9(1):114. Doi: https://doi.org/10.1186/s13613-019-0591y.

16. Martínez GH, Rodriguez ML, Vaquero MC, Ortiz R, Masclans JR, Roca O, et al. High-Flow Oxygen with Capping or Suctioning for Tracheostomy Decannulation. N. Engl. j. med. [Internet]. 2020 [cited 2021 May 1];383(11):1009-1017. Doi: https://doi.org/10.1056/NEJMoa2010834 e

17. Girault C, Gacouin A. [Tracheotomy and high-flow oxygen therapy for mechanical ventilation weaning]. Rev Mal Respir [Internet]. 2017 Apr [cited 2021 May 1];34(4):465-476. Doi: https://doi.org/10.1016/j.rmr.2017.03.026

18. Pires P, Marques C, Masip J. Canulas nasais de alto fluxo: uma alternativa de oxigenoterapia na insuficiência respiratória aguda. Sociedade portuguesa de cuidados intensivos- 2018. http://revista.spmi.pt ; DOI: 10.24950/rspmi/revisao/240/2/2018

19. Subirana-Ferrés C, García-Hornos E, Ruiz-Barrio P, Díaz-Martos I, Córdoba-Izquierdo A. Beneficios y cuidados enfermeros en el uso de cánula nasal de alto flujo en el paciente con insuficiencia respiratoria aguda. Metas enferm. [Internet]. 2018 [cited 2021 May 1];21(8):508. Available from: http://search.ebscohost.com/login. aspx?direct=true\&db=lth\&AN=132616141\&lang=pt-br\&site=ehost-live

20. Coudroy R, Frat J-P, Ehrmann S, Pène F, Terzi N, Decavèle $M$, et al. High-flow nasal oxygen therapy alone or with non-invasive ventilation in immunocompromised patients admitted to ICU for acute hypoxemic respiratory failure: the randomised multicentre controlled FLORALI-IM protocol. BMJ Open. [Internet]. 2019 [cited 2021 May 1];9(8):e029798. Doi: https://doi.org/10.1136/bmjopen-2019$\underline{029798}$

21. Ling Sang, Lingbo Nong, Yongxin Zheng, Yonghao Xu, Sibei Chen, Yu Zhang, et al. Effect of high-flow nasal cannula versus conventional oxygen therapy and non-invasive ventilation for preventing reintubation: a Bayesian network meta-analysis and systematic review. J. thorac. dis. [Internet]. 2020 [cited 2021 May 1];12(7):3725-36. Doi: http://dx.doi.org/10.21037/jtd-20-1050

22. Abobakr M, Abdalla A, Barakat T, Abdel-Hady H. Implementation of a protocol-based strategy for weaning nasal high flow therapy in preterm infants. Pediatric pulmonology [Internet]. 2020 [cited 2021 May 1];55(12):3319-27. Available from: http://search.ebscohost.com/login.aspx?direct=true\&db=mdc\&AN=33034941\&lang=pt-br\&site=ehost-live

23. Birk R, Handel A, Stuck BA, Hormann K, Sommer JU. Heated air humidification vs. cold air nebulisation in tracheotomised patients increases cilia beat frequency in tracheal epithelium and reduces frequency of suctioning procedures. European respiratory journal [Internet]. 2016 [cited 2021 May 1];48. Doi: 10.1183/13993003.congress-2016.PA3751

24. Mendes RM, Nunes ML. A importância da enfermagem de reabilitação nas unidades de cuidados intensivos portuguesas. Rev Port Enf Reab [Internet]. 2018 [cited 2021 May 1];1(2):8-13. Doi: https://doi.org/10.33194/rper.2018.v1.n2.01.4406

25. Ferreira MDF, Peres M. Impementação de um programa de reabilitação: intervenção do enfermeiro especialista de reabilitação numa UCI. Rev Port Enf Reab [Internet]. 2020 [cited 2021 May 1];3(2):68-75.

26. Yagi M, Morita K, Matsui H, Michihata N, Fushimi K, Koyama T, et al. Outcomes After Intensive Rehabilitation for Mechanically Ventilated Patients: A Nationwide Retrospective Cohort Study. Arch. phys. med. rehabil. [Internet]. 2021 Feb;102(2):280-289. doi: 10.1016/j.apmr.2020.09.389

27. Ponfick M, Wiederer R, Bösl K, Neumann G, Lüdemann-Podubecka J, Gdynia HJ, Nowak DA. The influence of weaning duration on rehabilitative outcome in early neurological rehabilitation. NeuroRehabilitation [Internet]. 2014 [cited 2021 Apr 29]2014;34(3):493-8. Doi: 10.3233/NRE-141066. PMID: 24473250 


\section{(c) $(7) \ominus$}

Este trabalho encontra-se publicado com a Licença Internacional Creative Commons Atribuição-NãoComercial-SemDerivações 4.0.

Direitos de Autor (c) 2021 Revista Portuguesa de Enfermagem de Reabilitação 